\title{
Preload Effect Elimination Technique for Piezoelectric Force Touch Sensing in Human-Machine Interactivities
}

\author{
Jiahui Shi, Junliang Chen, Jingjing Lin, and Shuo Gao, Member, IEEE
}

\begin{abstract}
Piezoelectric touch sensing in interactive displays gains increasing attentions due to its high force detection sensitivity and intrinsic mechanical-to-electrical conversion ability. However, the instable force-voltage responsivity induced by preload effect of piezoelectric materials reduces the force detection accuracy of secondary force touches, which is important to touch and haptic applications such as peek-pop. To address this issue, in this article, we present a preload effect elimination technique, in which the relationship between the piezoelectric coefficients and static preload is studied first, and then the detected secondary force touch is calibrated by using the previously applied static force information. Experimental results demonstrate that the force detection accuracy is boosted by $15.17 \%$ after applying the developed technique to secondary force touches with different preload values, potentially allowing the system to precisely interpret secondary force touch amplitude and hence enhancing the development of touch sensing in interactive displays.
\end{abstract}

Index Terms-Piezoelectric devices, preload effect, high detection accuracy, interactive displays

\section{INTRODUCTION}

$\mathrm{T}$ OUCH panels are ubiquitously utilized in modern electronic devices, ranging from consumer products [1][2][3] to medical applications [4][5][6]. Conventional touch interfaces locate users' touch points by capacitive and resistive means [7][8]. However, along with development of electric and information technologies, recognizing force amplitude is craved in plenty of scenarios since the highly efficient Human-Machine Interactivity (HMI) is essential for many applications such as fine haptic feedback [9][10]. The detection of force amplitude can make the interactivity between Apps and users close to reality and help developers exploit various functions based on different force levels. Hence, integration of force sensing into HMI systems gains considerable attentions.

Among various force sensing techniques, piezoelectric-based architectures offer high detection sensitivity, low power consumption and simple panel structure

Submitted July 12020 . This work was supported in part by the National Natural Science Foundation under Grant 61803017 and Grant 61827802, and in part by the Beihang University under Grant KG12090401 and Grant ZG216S19C8.

Jiahui Shi, Junliang Chen, Jingjing Lin, and Shuo Gao are with the School of Instrumentation Science and Optoelectronic Engineering, Beihang University, Beijing 100191, China (e-mail: shuo_gao@buaa.edu.cn). compared with its counterparts, hence triggering global interests [11][12]. Fruitful and systematic research results have been reported in literature. In [12], authors theoretically analyzed the mechanical properties of piezoelectric touch panels. It was proved that piezoelectric technique could provide higher detection sensitivity than the commercialized capacitive architecture. Nevertheless, successful use of piezoelectric touch panels in commercial products is rare. One key limitation is the instable force-voltage responsivity [13][14], which indicates that the same force amplitude can induce distinct electric outputs, hindering detection accuracy. The instable responsivity originates from touch panels' mechanical boundary conditions, piezoelectric materials' force detection mechanisms and user's touch behaviors.

Although remarkable progress has been made in stabilizing force-voltage responsivity [15][16][17], some factors still challenge high detection accuracy, one of which is the preload effect of piezoelectric material [18]. When piezoelectric materials are under static force, the status of molecular structures is affected, and hence influencing the ability of mechanical-to-electrical conversion, indicating the change of values of piezoelectric coefficients [19]. The preload effect is widely used to tune a piezoelectric sensor's resonant frequency [20]. However, when referring to touch detection, an instable force sensing responsivity issue emerges when secondary force touch events are performed. When a user first applies a static force touch, and then carries out a further force touch e.g., secondary force touch for the peek-pop function in iPhones, the piezoelectric coefficient of the secondary force touch is altered due to the existence of the previously applied static force touch. Therefore, the detection accuracy for the secondary force touch is reduced. This will hinder the sensitivity of the machine to provide haptic feedback, such as the idea of "active click" [21] and weaken the user's interactive experience.

To address this issue, in this article, we present a calibration method to reduce the influence of preload effect. Here, the relationship between preload and piezoelectric $d_{33}$ coefficient is established experimentally first, and then an algorithm is developed to calibrate the force-voltage responsivity for secondary force touches. Finally, an improved detection accuracy of $15.17 \%$ is obtained.

This article is structured as follows. Section II presents the experimental testbed to formulate the preload effect of the piezoelectric device with a piezoresistive device. Section III 


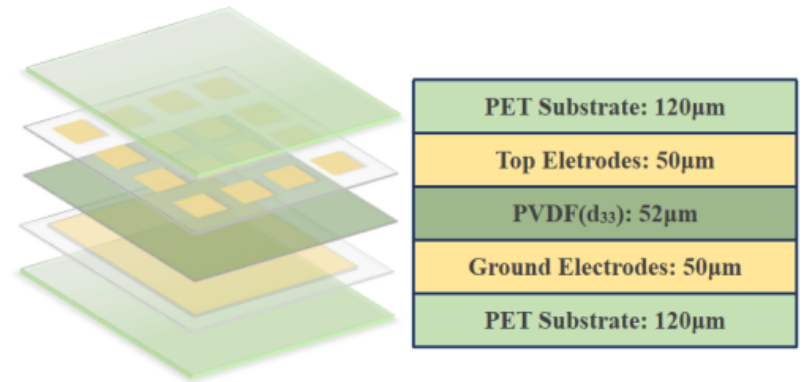

(a)

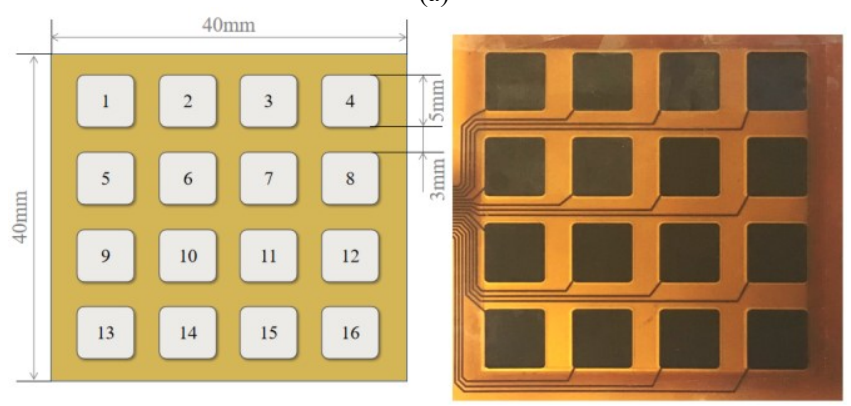

(b)

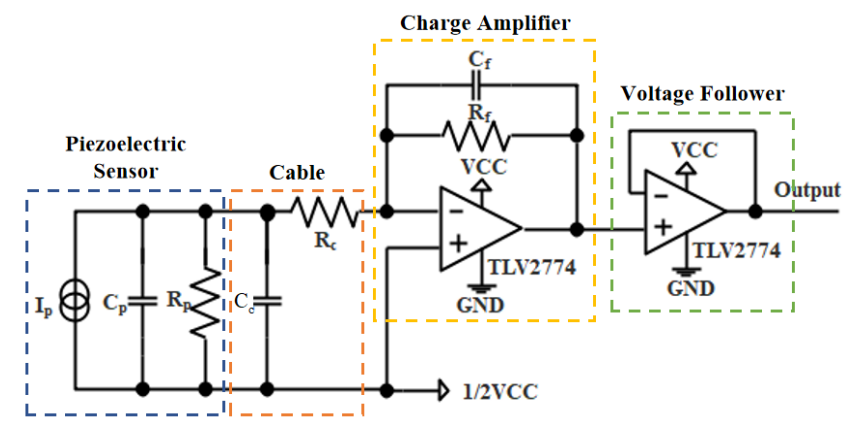

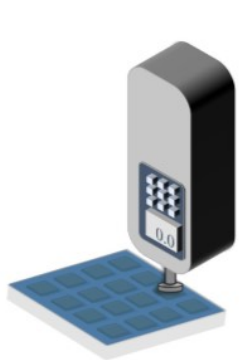

(d)

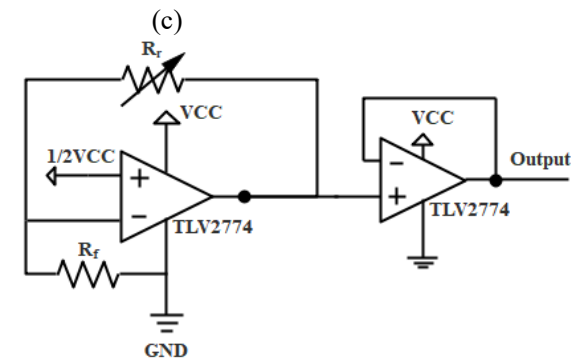

(e)
Fig.1. (a) Architecture of the touch panel. (b) Pad array of the touch panel. (c) Piezoelectric device readout circuit. (d) Berlincourt direct measurement. (e) Piezoresistive device readout circuit.

proposes a technique to eliminate the preload effect. Section IV discusses the characteristic of piezoelectric touch panel and the results of applying the proposed preload effect elimination algorithm, and finally, our conclusions are presented in section V.

\section{EXPERIMENTAL TESTBED}

To diminish the effect of preload and improve the detection accuracy for secondary force touches, our proposed technique is based on the examination of the relationship between preload and force detection responsivity. In this section, we will first describe the fabrication of a piezoelectric touch panel, and then, demonstrate the experimental study to verify the relationship utilized piezoresistive device.

\section{A. Piezoelectric Touch Panel Fabrication}

A piezoelectric touch panel is fabricated as part of the testbed (shown in Fig. $1 \mathrm{a}$ and b). The touch panel consists of 5 layers, which are PET (Polyethylene Terephthalate)/Cu (Patterned Electrodes)/PVDF (Force Sensing Layer) $/ \mathrm{Cu}$ (Ground Electrode)/PET from top to bottom. In the layer of patterned electrodes, $5 \times 5 \mathrm{~mm}^{2}$ square copper sheets are arranged in a $4 \times 4$ array, working as sensing electrodes. The ground electrode is a continuous $\mathrm{Cu}$ sheet. PVDF film is commercially purchased from Solvey Corp (UK).

To collect force induced charges, a charge amplifier-based circuit is assembled. The circuit diagram is provided in Fig. $1 \mathrm{c}$. By carrying out Berlincourt direct measurement method [22] with a commercial force sensor as Fig. $1 \mathrm{~d}$ shows. In this method, a dynamic force $F$ is applied parallel to the polarization and the resulting charge $Q$ is collected from the same surfaces on which the force applied. The longitudinal piezoelectric coefficient is defined as (1):

$$
d_{33}=Q_{\max } / F_{\max }
$$

Thus, detection sensitivity and responsivity of the fabricated piezoelectric touch panel can be obtained.

\section{B. Testbed Establishment}

To precisely measure applied preload at a set position, a commercial piezoresistive force sensor is employed. Resistance change of the piezoresistive force sensor can reflect the amplitude of the preload in real-time. The readout circuit of piezoresistive device is depicted in Fig. 1 e.

The experimental testbed is fabricated as Fig. 2 a. Considering that the force sensor needs to be stably attached to the piezoelectric touch panel, the piezoelectric touch panel and the piezoresistive force sensor together with the force delivering object are clipped between two pieces of acrylic plates. The two acrylic plates are drilled at four corners and can be screwed at desired levels to provide different preload amplitudes. A steel cylinder is used as the force delivering object to mimic user's finger touch and maintain the preload. After the well preparation of preload setup, force events are applied at the surface of the upper acrylic plate. A force shaker is utilized to output controlled force signals. Note that, the piezoresistive device detects both the force applied to the tested location of the touch panel and the static preload.

The dynamic force detected by the piezoelectric device $\left(F_{e}\right)$ can be expressed as (2):

$$
F_{e}=Q / d_{33}
$$

where $Q$ represents the applied force induced charges, while the force detected by the piezoresistive device $\left(F_{r}\right)$ can be expressed as (3):

$$
F_{r}=a \cdot \exp (c \cdot R V)+b \cdot \exp (d \cdot R V)
$$




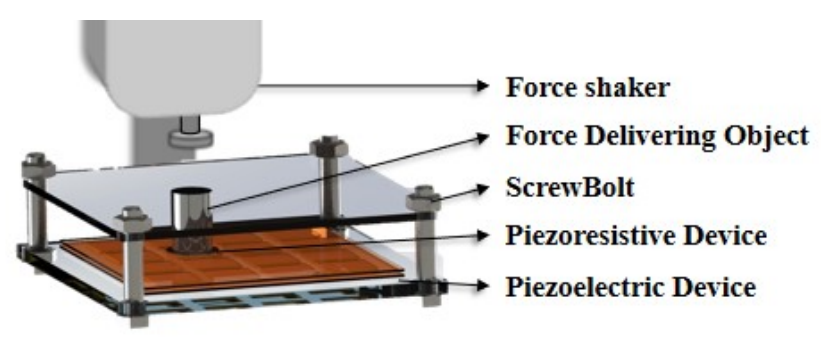

(a)

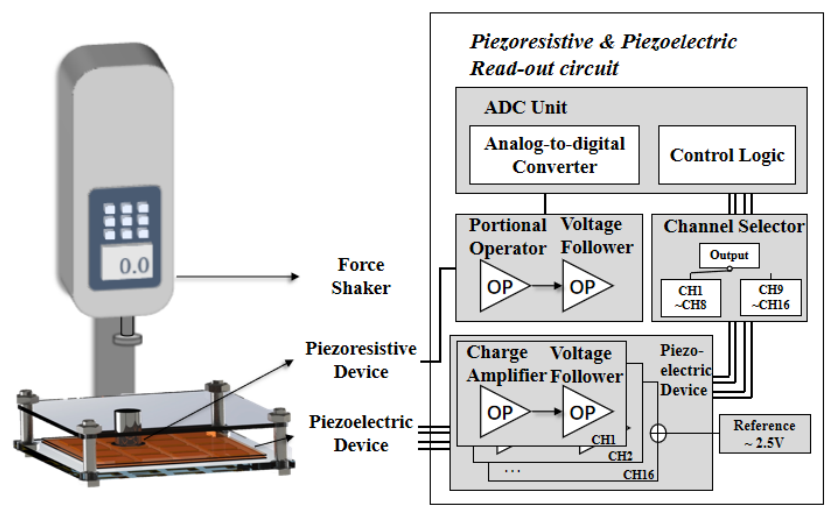

(b)

Fig.2. (a) The experimental testbed. (b) The testbed and readout circuitry.

where $R V$ represents the resistance value when the force is applied to the piezoresistive device, $a, b, c$, and $d$ are constants which are experimentally determined as $54.7,6.5,-0.38$ and -0.079 .

Here, we have an assumption that the force value read from the piezoresistive sensor $\left(F_{r}\right)$ is equivalent to that on the surface of the piezoelectric touch panel $\left(F_{e}^{\prime}\right)$, which includes both dynamic component $\left(F_{e}\right)$ given by the force shaker and static component $\left(F_{\text {sta }}\right)$ determined by different levels of drilling, as they are closely attached to each other. the relationship can be suggested as:

$$
F_{r}=F_{e}^{\prime}=F_{e}+F_{s t a}
$$

Hence, the force value read from the piezoresistive sensor is a reliable reference to obtain the relationship between preload and piezoelectric force detection responsivity as well as to evaluate the accuracy of force detection based on piezoelectric device. In addition, the stability and thinness of the piezoresistive sensor make it a proper auxiliary detection device. The outputs from the two independent systems are synchronized to obtain the relationship, which is depicted in Fig. 2 b.

\section{Methodology}

In this section, we will present two algorithms. The first is used to detect the preload applied to the piezoelectric touch panel, while the second is the preload elimination method established on the correlation between the preload and piezoelectric $d_{33}$ coefficient. Note that in the second one, the

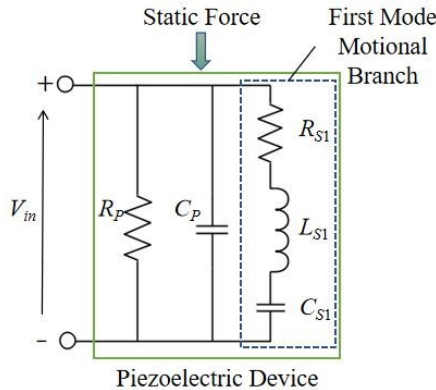

(a)

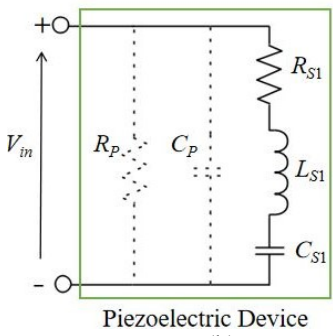

(b)

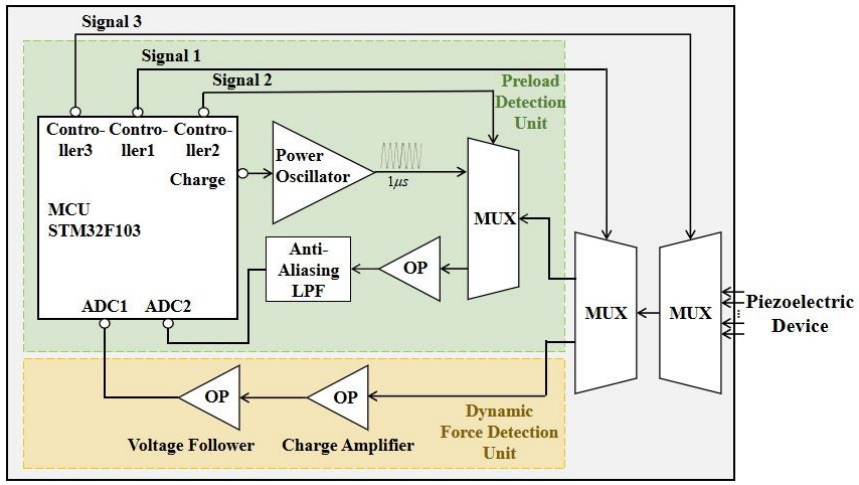

(c)

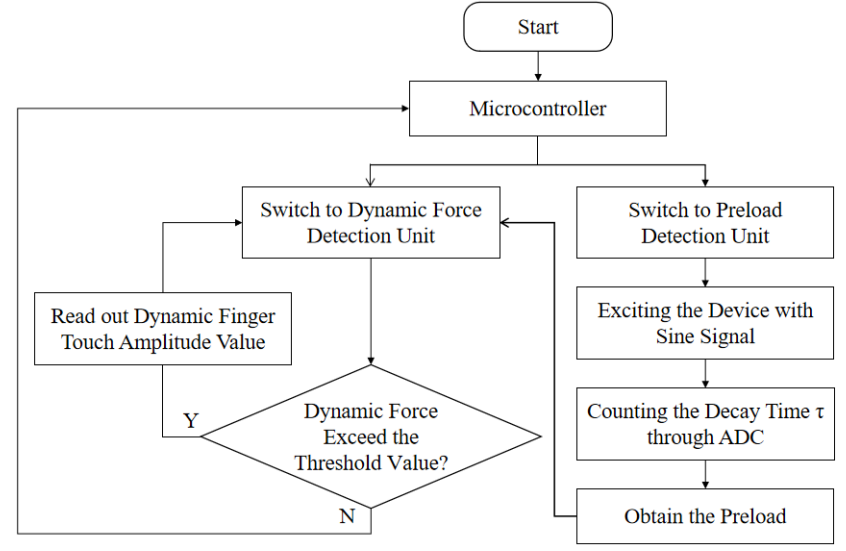

(d)

Fig.3. (a) The equivalent model of piezoelectric device in first resonance mode. (b) Effective circuit during free oscillation phase. (c) The readout circuitry for both dynamic and static force sensing. (d) Flow chart of the preload effect elimination algorithm.

preload is determined by a commercial piezoresistive sensor. Nevertheless, in a practical touch sensing scenario, only the piezoelectric touch panel is employed. Therefore, the first algorithm is necessary to interpret the applied preload in real situations.

\section{A. Preload Detection Method}

As the amplitude of static force touch is different within the users, to remove the effect of preload, an essential step is to detect the static force, which is treated as the preload applied from the user. Here, the change of resonant characteristics of piezoelectric device [23] is employed to detect the preload. It can be explained as the following demonstration. The equivalent model of piezoelectric device can be modelled by the Extended Butterworth Van Dyke circuit in first resonance 
as Fig. 3 a [24], which consists of a dielectric input capacitance $C_{p}$, a leakage resistance $R_{p}$, and a first resonance mode branch $\left(R_{s l}, C_{s l}, L_{s l}\right)$. This branch can be expressed with complex components $\left(C_{s l}, L_{s l}{ }^{\prime}\right)$. The components can be determined as (5), (6) [25]:

$$
\begin{gathered}
C_{s 1}^{\prime}=\left[\left(\varepsilon_{33} A / t\right) /\left(1-k_{t}^{2}\right)\right] \cdot\left[\left(f_{p}^{2}-f_{s}^{2}\right) / f_{p}^{2}\right] \\
L_{s 1}^{\prime}=1 /\left(4 \pi^{2} f_{s}^{2} C_{s 1}^{\prime 2}\right)
\end{gathered}
$$

where $t$ represents thickness of the piezoelectric layer, $k_{t}$ represents the electromechanical coupling constant, $f_{p}$ represents the parallel resonance frequency, and $f_{s}$ represents the series resonance frequency.

If the device is excited by a sinusoidal voltage signal, whose frequency is in the vicinity of the resonance frequency, the time sequence will have two phases: the excitation phase and the free oscillating phase. The applied signal $V_{\text {in }}$ is set to begin and end with zero value, so $\mathrm{C}_{\mathrm{p}}$ is shorted at the end of the excitation phase, and the model in Fig 3 a can be simplified as Fig. 3 b shows. During the free oscillating phase, the decay time $\tau$ can be determined in (7):

$$
\tau=2 L_{s 1} / R_{s 1}
$$

$R_{s l}$ and $L_{s l}$ will change with different applied force, so the decay time $\tau$ is related with the preload value. It can be calculated as (8):

$$
\tau=\left(t_{2}-t_{1}\right) / \ln \left(i\left(t_{1}\right) / i\left(t_{2}\right)\right)
$$

Where $i(t)$ represents the current value at time $t$. Then, the static force $\left(F_{\text {sta }}\right)$ can be calculated by the decay time, $\tau$, as $(9)$ :

$$
F_{\text {sta }}=a_{1} \cdot \exp \left(a_{2} \cdot \tau\right)
$$

where $a_{1}$ and $a_{2}$ are constants modified by experiments.

To obtain the static force, a system is assembled. The circuit diagram of the piezoelectric device preload detection unit is depicted in Fig. 3 c. The preload detection unit consists of a microcontroller (MCU), a multiplexer (MUX), a lower-pass filter (LPF) and a power oscillator. First, the piezoelectric touch panel is connected to the detection unit, which will be excited by a sinusoid signal generated by the power oscillator. Then the piezoelectric touch panel is connected to the analog-to-digital converter (ADC) and read out the discharging time, through which the preload can be interpreted according to (9).

\section{B. Preload Elimination Method}

To eliminate the preload effect, we introduce a correction factor $\alpha$. The relationship between the detected force $F$ and the calibration force $F^{\prime}$ can be presented as (10):

$$
F^{\prime}=\alpha \cdot F
$$

The correction factor $(\alpha)$ can be expressed as (11):

$$
\alpha=F^{\prime} / F=R / R_{p}
$$

where $R$ represents the responsivity without preload and $R_{p}$ represents the responsivity with a specific preload, which can be expressed with preload value.

The preload detection unit and the dynamic force detection unit are both connected to the touch panel as depicted in Fig. $3 \mathrm{c}$. To obtain the correction factor and achieve the preload elimination, an algorithm is designed as shown in Fig. $3 \mathrm{~d}$. The whole process is carried in the following way: first, force touch events are applied to the piezoelectric device and the microcontroller generates Signal 1 to control the switch between the preload detection unit and the dynamic force detection unit. Once the switch is connected to the preload detection unit, the unit works, and the preload can be obtained. According to the preload, the correction factor $\alpha$ would be available. Then, the dynamic force detection unit is connected to the piezoelectric device and captures the output voltage amplitude. There is a threshold value to decide if the unit will keep working. The correction factor $\alpha$ is utilized to ensure the force sensing responsivity is maintained in preload scenarios. To describe the accuracy of the method, piezoresistive device is attached closely to the piezoelectric device to obtain the real-time force applied to the device. The experimental results will be demonstrated in the following section.

\section{RESULTS AND DISCUSSION}

In this section, the characteristic of piezoelectric touch panel will be discussed, and the results of applying the proposed preload effect elimination algorithm are given.

\section{A. Characteristic of Piezoelectric Touch Panel}

Sensitivities and responsivities of the piezoelectric device are depicted in Fig. 4 a and b. Sensitivities suggest the minimum signal amplitude that can detect from one piezoelectric based touch panel, while responsivities suggest the ratio of signal voltage to applied force. The fabricated touch panel provides an average force sensing sensitivity and responsivity of $48.9 \mathrm{mN}$ and $298 \mathrm{mV} / \mathrm{N}$, respectively. The responsivity $R$ is expected to be maintained for secondary force touches. The experimental results of the system average responsivity under different preload are given in Fig. 4 c, which shows that the force-voltage responsivity decreases with the increment of preload amplitude. The correction factor is modeled as (12):

$$
\alpha=R /(m \cdot P L+n)
$$

where $P L$ indicates the preload value, $m$ and $n$ are constants which are experimentally determined as -0.1572 and 0.3059 . The goodness of this fit is 0.9867 . This relationship will be used to calibrate secondary force events under different preload values. 


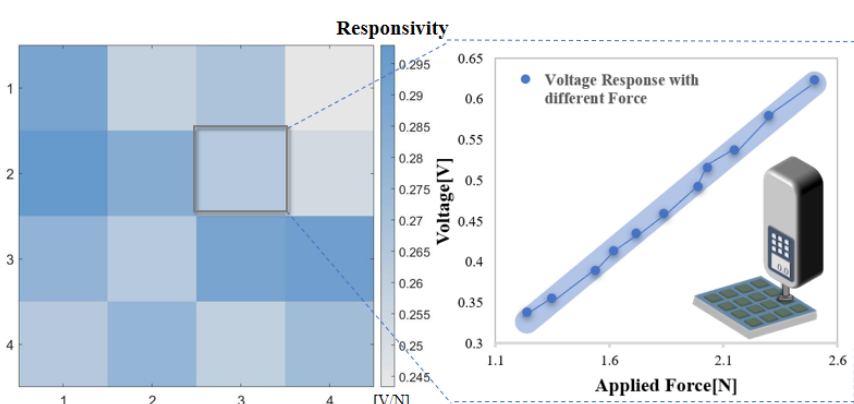

(a)

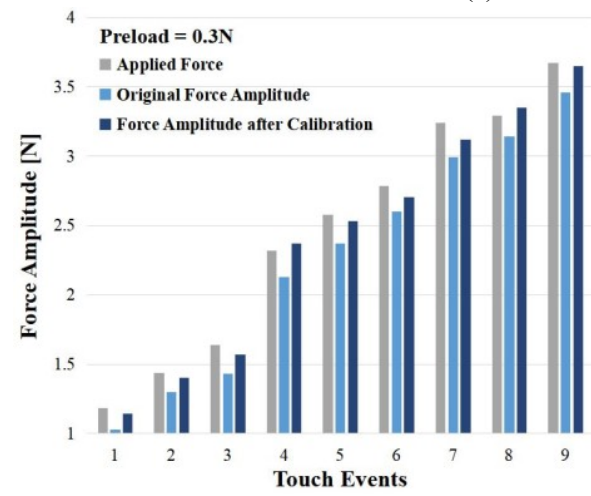

(d)

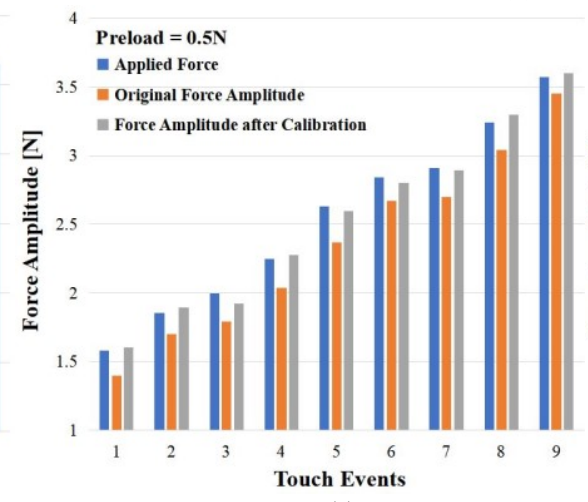

(e)

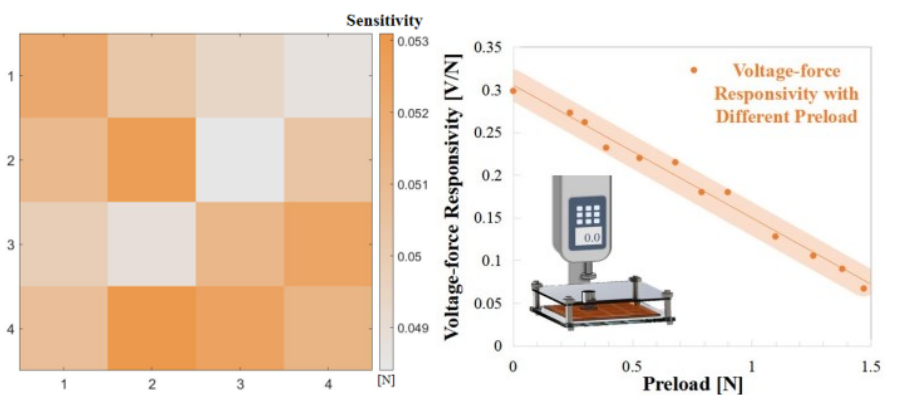

(c)

Fig.4. (a) Responsivities of the piezoelectric device. (b) Sensitivities of the piezoelectric device. (c) The relationship between preload and force-voltage responsivity of the piezoelectric touch panel. (d-f) The comparisons of original force amplitudes, calibrated force amplitudes and applied force amplitudes under preload of $0.3 \mathrm{~N}, 0.5 \mathrm{~N}$ and $0.8 \mathrm{~N}$.

\section{B. Force Touch Calibration}

Comparisons of user applied force amplitudes at a specific piezoelectric sensor and corresponding calibration and calibration-free force amplitudes, under preload of $0.3 \mathrm{~N}, 0.5 \mathrm{~N}$ and $0.8 \mathrm{~N}$, are shown in Fig. $4 \mathrm{~d}$, e, and f. It can be observed that after calibration, the force amplitudes get closer to the user applied force amplitudes than their counterparts, indicating the preload effect is eliminated efficiently. The average improved detection accuracy is calculated from the deviation decrement before and after calibration by averaging results from three pads, which are pad 3, 4, and 7 (labeled in Fig. 1 b). The reason to record these three pads is that the pad array is rotationally symmetric, and these pads represent the characteristic of boundary, corner and interior points, respectively. As Fig 5 depicts, when the preload values are $0.3 \mathrm{~N}, 0.5 \mathrm{~N}$ and $0.8 \mathrm{~N}$, comparing the deviation from the true value of calibration and calibration-free results, the average improved detection accuracy with calibration are $13.87 \%, 15.69 \%$ and $15.96 \%$, respectively. It suggests that the detection accuracy of secondary force touch increases $15.17 \%$ in average by the implemented technique. The presented technique successfully eliminates the effect of preload, boosting the detection accuracy of piezoelectric touch panel.

Evaluation of computational time cost is significant for real-time applications. The computational time cost consists of the preload detection time and secondary force detection time slot. For the former, the time budget is mainly consumed by counting the discharge time. Based on (7), approximately less than $300 \mu \mathrm{s}$ is required, which is neglectable comparing to the interval between user performed static and secondary force touches. The force calibration algorithm can be completed within ms-level time, therefore the time delay of the methodology is acceptable and feasible.

\section{CONCLUSION}

In this article, a force-voltage responsivity stabilization technique which eliminates the influence of preload effect for piezoelectric touch panels is developed. Experimental results demonstrate that the preload effect is successfully removed by giving an average improved detection accuracy of $15.17 \%$. The technique presented in this article allows the system to precisely interpret user applied secondary force touch, enhancing user's experience and therefore prompting the development of piezoelectric force touch sensing in interactive displays to provide more precise haptic feedback.

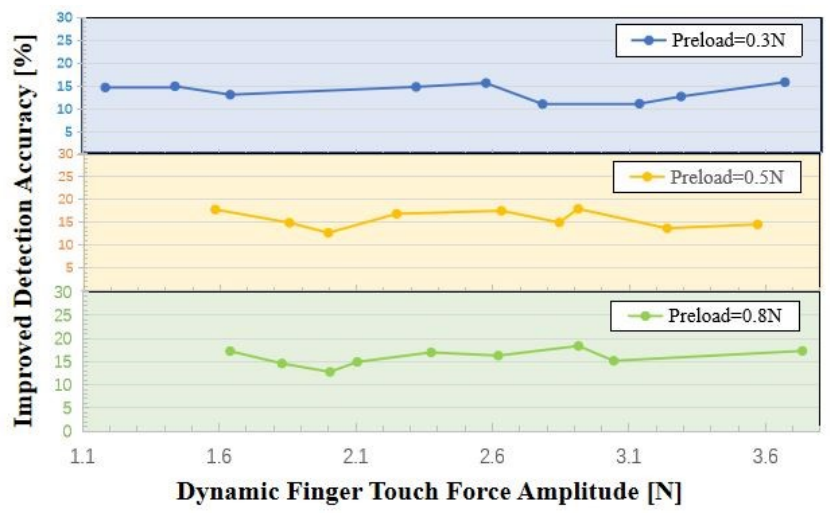

Fig.5 Improved detection accuracy under different preload values with varied dynamic finger touch amplitude. 


\section{REFERENCES}

[1] L. Du et al., "A single layer 3-D touch sensing system for mobile devices application," IEEE Transactions on Computer-Aided Design of Integrated Circuits and Systems, vol. 37, no. 2, pp. 286-296, 2018.

[2] C. Wen and C. Huang, "A paperless fax machine with a single-touch panel," IEEE Transactions on Consumer Electronics, vol. 54, no. 4, pp. 1488-1491, 2008.

[3] C. Lin, C.S. Li, Y. Chang, YC.C. Hung, and A. Lin, "3D Stylus and pressure sensing system for capacitive touch panel," 2012 IEEE International Conference on Consumer Electronics (ICCE), pp. 215-216, 2012.

[4] T. Kurata et al., "The usefulness of a simple computerized touch panel-type screening test for Alzheimer's disease patients," 2012 ICME International Conference on Complex Medical Engineering (CME), pp. 215-217, 2012.

[5] A. Cebrian, J. Millet, and I. Garcia, "Flat panel displays for medical monitoring systems," 2001 Conference Proceedings of the 23rd Annual International Conference of the IEEE Engineering in Medicine and Biology Society, vol. 4, pp. 3966-3969, 2001.

[6] N. Morimoto, T. Kurata, K. Sato, Y. Ikeda, K. Abe, and S. Sato, "A computerized touch panel-type screening test in patients with motor neuron disease," 2012 ICME International Conference on Complex Medical Engineering (CME), pp. 360-363, 2012.

[7] P. T. Krein and R. D Meadows, "The electroquasistatics of the capacitive touch panel," IEEE Transactions on Industry Applications, vol. 26, no. 3, pp. 529-534, 1990.

[8] C. Lai and C. Tsai, "Neural calibration and Kalman filter position estimation for touch panels," Proceedings of the 2004 IEEE International Conference on Control Applications, vol. 2, pp. 1491-1496, 2004.

[9] S. D. T. Kelly, N. K. Suryadevara, and S. C. Mukhopadhyay, "Towards the implementation of IoT for environmental condition monitoring in homes," IEEE Sensors Journal, vol. 13, no. 10, pp. 3846-3853, 2013.

[10] S. Jo, H. Cho, and H. Yoo, "A fully reconfigurable universal sensor analog front-end IC for the internet of things era," IEEE Sensors Journal, vol. 19, no. 7, pp. 2621-2633, 2019.

[11] S. H. Bae, O. Kahya, B. K. Sharma, J. Kwon, H. J. Cho, B. Ozyilmaz, and J. H. Ahn, "Graphene-P (VDF-TrFE) multilayer film for flexible applications," ACS Nano, vol 7, no. 4, pp. 3130-3138, 2013.

[12] S. Gao, V. Arcos, and A. Nathan, "Piezoelectric vs. capacitive based force sensing in capacitive touch panels," IEEE Access, vol. 4, pp. 3769-3774, 2016.

[13] S. Gao, X. Wu, H. Ma, J. Robertson, and A. Nathan, "Ultrathin multifunctional graphene-PVDF layers for multidimensional touch interactivity for flexible displays," ACS Applied Materials \& Interfaces, vol. 9, no. 22, pp. 18410-18416, 2017.

[14] S. Gao and L. Wu, "Why piezoelectric based force sensing is not successful in interactive displays?" IEEE Consumer Electronics Magazine, vol. 4, no. 8, pp. 28-31, 2019.

[15] S. Gao, J. Duan, Z. Wei, and A. Nathan, "P-193: High force sensing accuracy in piezoelectric based interactive displays by artificial neural networks," Wiley Online Library, vol. 49, pp. 1893-1896, 2018.

[16] S. Gao, J. Duan, V. Kitsos, D. R. Selviah, and A. Nathan, "User-oriented piezoelectric force sensing and artificial neural networks in interactive displays," IEEE Journal of the Electron Devices Society, vol. 6, pp. 766-773, 2018.

[17] A. Huang, S. Gao, Y. Dai, V. Kitsos, W. Tian, and L. Xu, "A capacitive information based force-voltage responsivity stabilization method for piezoelectric touch panels," IEEE Journal of the Electron Devices Society, vol. 7, pp. 1018-1025, 2019.

[18] J. Wooldridge, A. Muniz-Piniella, M. Stewart, T. A. V. Shean, P. M. Weaver, and M. G. Cain. "Vertical comb drive actuator for the measurement of piezoelectric coefficients in small-scale systems." Journal of Micromechanics and Microengineering, vol. 3, no. 23, pp. 28-33, 2013.

[19] S. Gao and A. Nathan. "A flexible multi-functional touch panel for multi-dimensional sensing in interactive displays," Cambridge University Press, 2019.

[20] P. Smithmaitrie, P. Suybangdum, P. Laoratanakul, and N. Muensit, "Design and performance testing of an ultrasonic linear motor with dual piezoelectric actuators," IEEE Transactions on Ultrasonics, Ferroelectrics, and Frequency Control, vol. 59, no. 5, pp. 1033-1042, 2012.

[21] M. Fukumoto and T. Sugimura, "Active Click: Tactile Feedback for Touch Panels", Proc. Conf. Extended Abstracts on Human Factors in Computing Systems (CHI '01 ), pp. 121-122, 2001.
[22] D. Berlincourt, and H. H. A. Krueger . "Domain Processes in Lead Titanate Zirconate and Barium Titanate Ceramics." Journal of Applied Physics, vol. 30, no. 11, pp. 1804-1810, 1959.

[23] S. Ozeri and D. Shmilovitz, "Static force measurement by piezoelectric sensors," in 2006 IEEE International Symposium on Circuits and Systems, 2006.

[24] A. Vives and Antonio, "Piezoelectric Transducers and Applications," 2004.

[25] S. Sherrit, H. D. Wiederick, B. K. Mukherjee, and M. Sayer, "An accurate equivalent circuit for the unloaded piezoelectric vibrator in the thickness mode," Journal of Physics D: Applied Physics, vol. 30, pp.: 2354-2363, 1997.

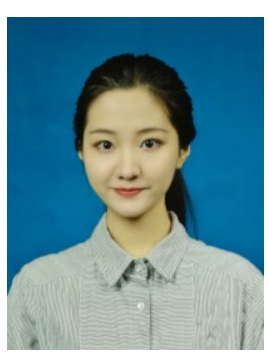

Jiahui SHI is a student at the School of Instrumentation and Optoelectronic Engineering, Beihang University, Beijing, China

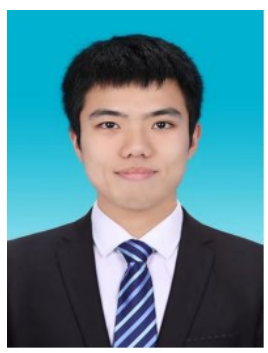

Junliang CHEN is a student at the School of Instrumentation and Optoelectronic Engineering, Beihang University, Beijing, China.

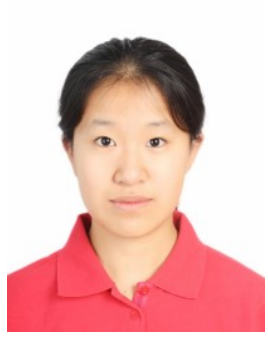

Jingjing LIN is a student at the School of Instrumentation and Optoelectronic Engineering, Beihang University, Beijing, China.

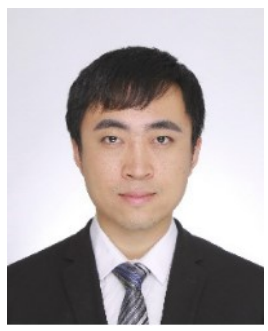

Shuo GAO received the Ph.D. in electrical engineering from the University of Cambridge, Cambridge, UK, in 2018. From 2017 to 2018, he was a Research Associate with the University College London, London, UK. He is currently an Associate Professor at the Beihang University, Beijing, China. His area of expertise is human-machine interactive systems. He has published over 50 articles as the first author, including peer-reviewed journals, flagship conferences, awarded US, UK and CN patents. He was a recipient of China Scholarship Council Ph.D. Student Fellowship in 2014. He serves on technical committees in various capacities. In terms of industrial experience, previously he worked as an optical fiber system engineer at Ciena Corporation, Ottawa, Canada, from 2012 to 2013; and a technique consultant at Cambridge Touch Technologies Inc., Cambridge, UK, from 2013 to 2017. 